\title{
Numerical modeling of pore size and distribution in foamed titanium
}

\author{
H. Shen ${ }^{\text {a }}$, S.M. Oppenheimer ${ }^{\text {b }}$, D.C. Dunand ${ }^{\text {b }}$, L.C. Brinson ${ }^{\mathrm{a}, \mathrm{b}, *}$ \\ a Mechanical Engineering Department, Northwestern University, 2145 Sheridan Road, Evanston, IL 60208-3111, USA \\ ${ }^{\mathrm{b}}$ Department of Materials Science and Engineering, Northwestern University, Evanston, IL 60208, USA
}

Received 10 December 2004; received in revised form 1 March 2005

\begin{abstract}
To facilitate the design and development of porous metals, simulation of their mechanical behavior is essential. As an alternative to complex tomography procedures, a methodology has been developed to construct a simulated microstructure that retains the essential features of the experimental material. The target material is a moderate porosity titanium foam that is being developed as a bone implant material. The methodology applies stereology theory to a foaming process based on growth of pressurized pores. Three-dimensional (3D) pore size and pore distribution information is derived from 2D sections for a sample with low porosity, early in the foaming process. A $3 \mathrm{D}$ microstructure is developed based on the $3 \mathrm{D}$ location and size distribution of the pores by use of a computational procedure. Pores are allowed to grow and coalesce in a simple simulated foaming process to achieve microstructures of higher porosity. These data have been used as inputs to write scripts of I-DEAS to create 3D finite element models which are then examined for basic global and local mechanical properties.
\end{abstract}

(c) 2005 Elsevier Ltd. All rights reserved.

Keywords: Titanium foam; Microstructure; Finite element; Pore size; Distribution

\section{Introduction}

Titanium and its alloys exhibit excellent mechanical and biological properties, thus making titanium-based foams attractive for bone-replacement implants in biomedical engineering (Dunand, 2004; Li et al., 2004; Wen et al., 2002b). In cellular mate-

\footnotetext{
* Corresponding author. Address: Mechanical Engineering Department, Northwestern University, 2145 Sheridan Road, Evanston, IL 60208-3111, USA. Tel.: +1 847467 2347; fax: +1 8474913915.

E-mail address: cbrinson@nwu.edu (L.C. Brinson).
}

rials, stiffness drops with the square of relative density (Gibson and Ashby, 1997), so porous titanium is expected to show a much reduced stress shielding effect as compared to current monolithic metallic implants exhibiting stiffness values up to 10 times higher than the hosting bone. Additionally, porous implants promote a stable bone-implant interface by the immigration of bone-forming cells from the surrounding bone (Chang et al., 1996; Li et al., 2004; Long and Rack, 1998; Okazaki et al., 1998; Spoerke et al., in press; Wen et al., 2002a,b). However, the reduced stiffness and the improved interface might be accompanied by reduced static or 
fatigue strength and ductility, as the result of stress concentrations near pores leading to intense local slip bands and pore wall bucking. To predict microscopic stress and strain localizations as well as macroscopic responses, finite element (FE) analyses providing full-field solutions are useful. FE simulations can aid in understanding the relationship between pore morphology, overall properties and strain localization. Ultimately the results can guide the designed processing of titanium foams with optimal properties for a given application. To obtain high-quality predictions of porous material response, it is critical to construct FE models that provide a reasonably accurate depiction of the actual geometry of the microstructure.

Many authors have used the FE method to model the mechanical properties of metallic foams, both in the elastic (Segurado and Llorca, 2002; Sihn and Roy, 2004; Thelen et al., 2004) and plastic ranges (Benke and Weichert, 2003; Li et al., 2004) of deformation. In particular, Li et al. (2004) simulated the microstructure of porous materials as particle-reinforced metal matrix composites (PR MMCs) in which the included phase had a zero modulus. Two-dimensional (2D) finite element models were created in which all porosity was closed and the influence of the pore morphology on mechanical responses was studied. Segurado and Llorca (2002) simulated a porous material by 3D cubic models containing 30 non-overlapping identical spherical pores with volume fraction up to $50 \%$ to study the effect of porosity on elastic moduli. However, most commercially used PR MMCs have low volume fractions of reinforcements, because ductility is extremely low for PR MMCs with over 30 vol.\% particulates (McDanels, 1985). Therefore, most FE simulations of PR MMCs assume isolated particles with no impingement. In a different approach, Benke and Weichert modeled a random foam as a three-dimensional (3D) framework of slender struts to study the damage in metal foams (Benke and Weichert, 2003). This is similar to the model for cellular mechanics which is based on beam theory and cell wall structure (Gibson and Ashby, 1997). Following this line, Sihn and Roy (2004) characterized representative 3D idealized cell ligaments to correlate the microstructure of opencell carbon foams with bulk properties. These models based on simulation of ligaments and pore walls are suitable for open-cell foams with high porosities, typically above $70 \%$. However, the titanium foams considered for orthopedic implants have a porosity of $40-50 \%$ as an optimal range to provide reduced stiffness and sites for bone ingrowth while maintaining mechanical durability.

The titanium foam considered here was processed by the solid-state foaming technique which consists of two steps: first, individual high-pressure argon pores are created within a billet by densification of powders in the presence of argon gas; a second foaming step consists of expansion, by plastic deformation of the surrounding matrix, of these individual pores at elevated temperature (Dunand, 2004; Murray and Dunand, 2003). The foamed microstructure, as shown in Fig. 1, is quite complex. The porosity of $40-50 \%$ leads to the coexistence of small pores and large pores. The large pores have a complex, tortuous shape which developed during the foaming step by coalescence of initially-isolated pores clustered around prior metallic powders (Murray and Dunand, 2003). Therefore, the previous two types of models which focus on simulating closed pores or ligaments are not appropriate to represent the microstructure of the target titanium foam.

Some researchers have developed models based on actual microstructures. Thelen et al. (2004) created 2D models based on metallographic crosssections of titanium foam. A 3D microstructure can be reconstructed by digitally stacking such sections. 3D microstructural images were obtained for PR MMCs by this technique and the experimental procedure was described in detail by $\mathrm{Li}$ et al. (1998). Shan and Gokhale (2001) created a 3D finite element model based on this serial sectioning method to study the stress and strain distribution of cast $\mathrm{Al}-\mathrm{Si}-\mathrm{Mg}$ base alloy containing pores. The

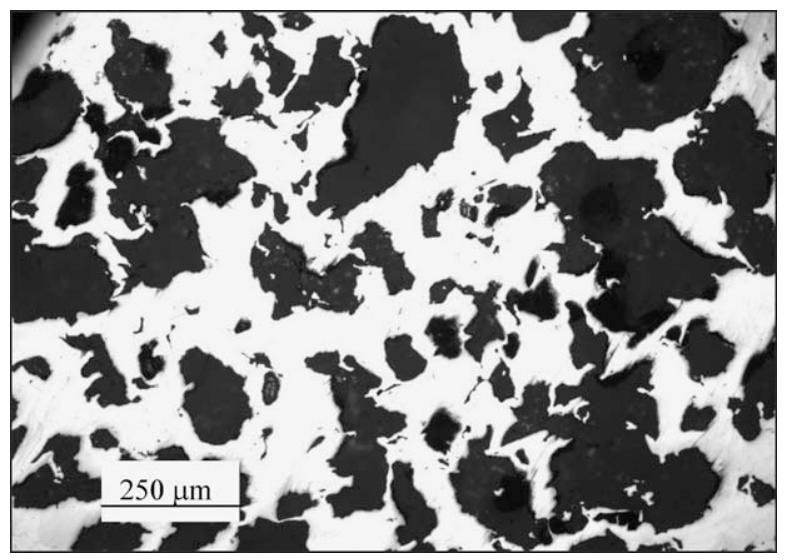

Fig. 1. Optical micrograph of metallographic cross-section for titanium foam with $50 \%$ porosity, showing small pores coexisting with large, interconnected pores with ragged morphology. 
porosity is very low for their material and the microstructure is relatively simple compared with typical metallic foams. However, this method is time-consuming and the accuracy is questionable given a typical pore sizes below $100 \mu \mathrm{m}$ for titanium foams. In addition, for titanium foams with $40-50 \%$ porosity, the specimens need to be vacuum-infiltrated with epoxy resin at regular intervals to minimize pore wall deformation and shape distortion during the sectioning process. These considerations make it impractical to create a $3 \mathrm{D}$ model for the material using serial sectioning method. Although 2D analysis seems practical using this method, as pointed by Shen and Lissenden (2002), 3D simulation is still necessary for studying local events.

In this paper, we develop a method to efficiently construct both 2D and 3D simulated models that capture the main features of the actual experimental material. At the early stage of foaming, when porosity was less than $25 \%$, pores are mostly rounded, generally equiaxed, and un-merged, as illustrated in Fig. 2(a) (Murray and Dunand, 2003). Metallographic cross-sections were prepared to collect 2D data of pore structure in this early foaming condition (Section 2). Then, 3D volumetric distribution of pores was simulated by the Saltykov method from 2D area distribution of pores in sections (Underwood, 1970). The microstructural distance

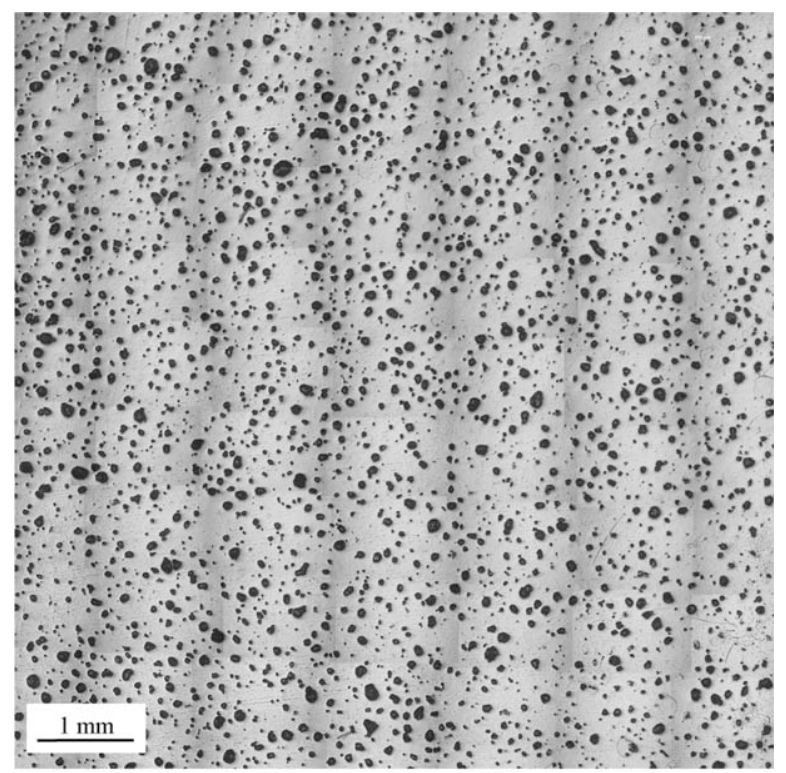

(a)

Fig. 2(a). Optical micrograph of metallographic cross-section for a titanium foam with $14.7 \%$ porosity, showing that pores are roughly spherical and isolated pores.

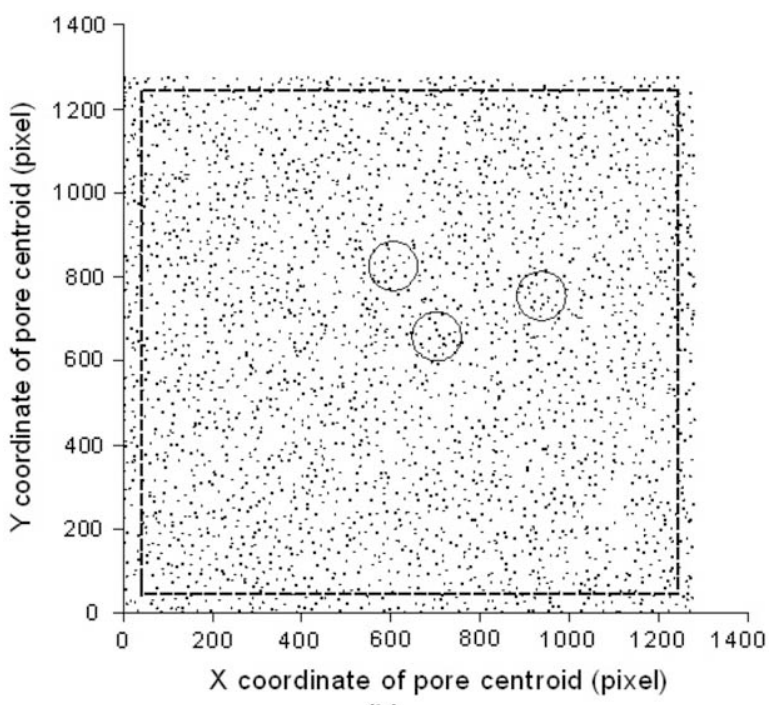

(b)

Fig. 2(b). Centroid distribution of pores in Fig. 2(a). Small circles are sample domains for the $K$ function to determine pore location distribution. The smaller dash line rectangular box is the section zone which is inset by distance $R$ from the edges of the full section to ensure that no circle falls outside the section.

distribution function was used to characterize the spatial distribution of pores based on several 2D sections (Section 3). Next, computer programs were developed to reconstruct the $3 \mathrm{D}$ microstructure at low porosity using the parameters obtained for the size and spatial distribution of pores based on these analyses. Then the microstructure of the foam at high porosity was achieved by a geometric simulation of pore growth and movement during foaming process (Section 4). By this method, the complex 3D microstructure of foam with porosity of $40-50 \%$ was obtained in a relatively simple, efficient, and accurate manner. This method, which applies stereology theory to the foaming process, can be considered as a new way to construct complex foam microstructure. A 3D finite element model was then created based on the simulated microstructure of the experimental material using the methodology (Section 5). The uniaxial stress-strain response was simulated and compared with measurements on titanium foams.

\section{Experimental procedures}

\subsection{Material fabrication}

Commercially available spherical titanium (CPTi) powders (from Starmet, Concord, MA) were 
sieved to a $149-177 \mu \mathrm{m}$ range, packed in a mild-steel can, backfilled with $3.3 \mathrm{~atm}$ argon and Hot Isostatic Pressed (HIPed) at $890^{\circ} \mathrm{C}$ for $120 \mathrm{~min}$ under $100 \mathrm{MPa}$ argon gas by Connaway Technologies and Isostatic Forging (Hilliard, OH). Cubic specimens with $9 \mathrm{~mm}$ edges were produced by electrodischarge machining and their surfaces were polished with 400 grit paper. Foaming was performed on these specimens in a high-vacuum furnace ( $p<10^{-5}$ Torr) by cycling temperature between 840 and $980^{\circ} \mathrm{C}$ with a period of $4 \mathrm{~min}$, corresponding to a heating/cooling rate slightly above $1 \mathrm{~K} / \mathrm{s}$. Such cycling about the allotropic point is known to induce superplasticity in titanium and to enhance the foaming kinetics (Davis et al., 2001; Dunand, 2004; Murray and Dunand, 2003, 2004a). Foaming was interrupted periodically by excursions to room temperature for specimen porosity determination. A measurement of the total porosity was performed by the Archimedes density method in distilled water, using a thin layer of vacuum grease to seal the surface and prevent water infiltration into the open porosity.

\subsection{Metallography}

The microstructural analysis was performed on a specimen of titanium foam of porosity less than $25 \%$. The mounted specimen was ground and polished using standard metallographic techniques down to a $0.05 \mu \mathrm{m}$ particle size. To obtain $2 \mathrm{D}$ information of pore size and distribution, three parallel sections were randomly chosen from the specimen. For each section, a series of at least 56 pictures were acquired with a magnification of $10 \times$ and subsequently combined to obtain an image of the whole section. These section images were used to acquire the location and distribution of 8634 pores Fig. 2(a) shows one such section and Fig. 2(b) the centroid distribution of the pores in it. The image was converted to a binary black and white image in order to measure centroid coordinates for analyzing the distribution pattern of pores. The large amount of data in each section image made pore size measurement difficult and so five smaller pictures were randomly selected from each section and used to measure the pore size. One such processed image is shown in Fig. 3. Since larger pores are more likely to intersect the edges of the field, a bias will be introduced for pore size measurement. To avoid the edge bias, a smaller box with four edges paralleled to the edges of the image was drawn excluding all the edge-touching pores as shown in Fig. 3. Pores intersecting the top and right edges of the box were counted in the measurement and pores intersecting the other two edges were excluded. Finally, 1664 pores were obtained for pore size characterization and the size was measured using commercial software (ImageJ 1.31v). The average pore area fraction is $14.7 \%$ which is used as the porosity for reconstructing a $3 \mathrm{D}$ microstructure.

\section{Characterization of size and location distributions of pores in the microstructure}

\subsection{Stereological analysis of size distribution}

Saltykov area analysis is a classical method to construct 3D inclusion volumetric distribution from 2D area distribution (Underwood, 1970). It is applicable not only to spherical inclusions but also, in principle, to convex ones in polydispersed systems. However, this method is limited to zero continuity

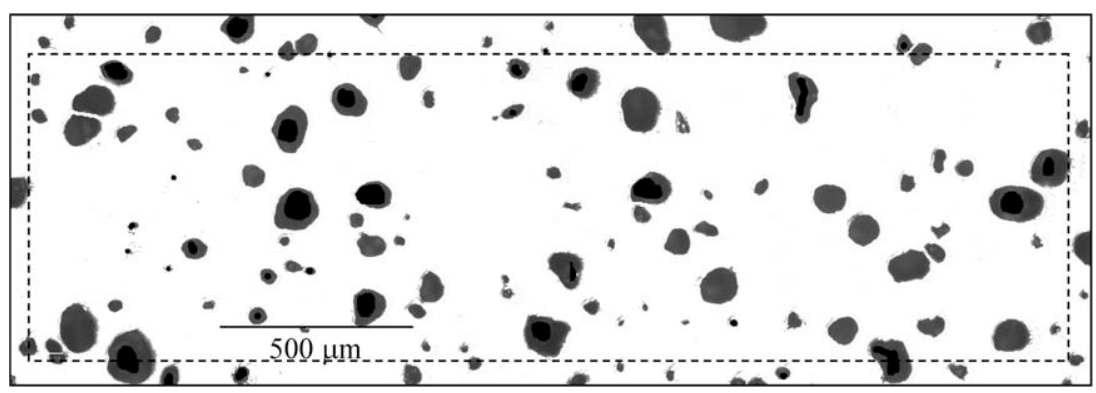

Fig. 3. An image for measuring pore size, cut from the larger section image (Fig. 2(a)), was converted to a binary black and white image To avoid the edge bias, a smaller dash line rectangular box was employed to exclude all the edge-touching pores. Pores intersecting the top and right edges of the box were counted and pores intersecting the other two edges were excluded. Five such images were cut from each of three sections for the pore size analysis. 
and low volume fraction of inclusions. In powder metallurgy, Liu et al. (1999) used this method to obtain initial guess for their iterative algorithm for 3D grain size distributions in liquid-phase sintered materials. Li et al. (1999) adopted this method to predict 3D distributions of particles in PR MMCs from known 2D information. The 3D size distribution predictions agreed rather well with the experimental 3D microstructural data although the predictions for particle shape and orientation were not promising. However, the shape and orientation for pores in the titanium foam need not be considered herein because of the approximate spherical shape of pores at porosity less than $25 \%$. Therefore, it is reasonable to adopt this stereological analysis for pore size characterization for the target material in the early foaming stages.

In the Saltykov area analysis, 2D section areas from metallographic images are studied and the pores in these sections are divided into $n$ size intervals. The number of pores in each interval is $N_{A}(i)$, where $i=1,2, \ldots, n$, and the corresponding diameter range for the $i$ th interval is $\left[D_{\max } \times\right.$ $\left.10^{-0.1(i-2)}-D_{\max } \times 10^{-0.1(i-1)}\right]$, where $D_{\max }$ is the diameter of the largest $2 \mathrm{D}$ pore in the examined sections. It is assumed that the diameter of the largest $2 \mathrm{D}$ pore is equal to that of the largest $3 \mathrm{D}$ spherical pore in the microstructure. Based on this assumption, the volumes of pores are also divided into the same number of identical intervals. With this assumption and the logarithmic scale for divisions of intervals, the 3D pore size distribution can be explicitly projected from the 2D size distribution and the formula for $n$ intervals is shown as follows (Liu, 1999):

$$
\begin{aligned}
N_{V}(j)= & \frac{1}{D(j)}\left[1.646121 \cdot N_{A}(i)-0.456123 \cdot N_{A}(i-1)\right. \\
& -0.11619 \cdot N_{A}(i-2)-0.041495 \cdot N_{A}(i-3) \\
& -0.017271 \cdot N_{A}(i-4)-0.007795 \cdot N_{A}(i-5) \\
& -0.003684 \cdot N_{A}(i-6)-0.00179 \cdot N_{A}(i-7) \\
& -0.000884 \cdot N_{A}(i-8)-0.000441 \cdot N_{A}(i-9) \\
& -0.000222 \cdot N_{A}(i-10)-0.000112 \cdot N_{A}(i-11) \\
& -0.000057 \cdot N_{A}(i-12)-0.000029 \cdot N_{A}(i-13) \\
& \left.-0.000015 \cdot N_{A}(i-14)-\cdots\right]
\end{aligned}
$$

where $N_{V}(j)$ is the number of pores in each volume interval, and $j=1,2, \ldots, n$.

From the metallographic study of the target titanium material, 1664 pores were obtained for pore size characterization from $2 \mathrm{D}$ sections. The maxi- mum diameter is $192.1 \mu \mathrm{m}$ and the minimum is $9.1 \mu \mathrm{m}$. The full range of diameters was divided into $n=14$ intervals for Saltykov area analysis. For each interval the size bin is $192.1 \times 10^{-0.1(i-2)} \mu \mathrm{m}$ to $192.1 \times 10^{-0.1(i-1)} \mu \mathrm{m}$, where $i=1,2, \ldots, 14$. The number of pores fitting in each interval was counted and a step plot of percentage distribution was plotted, as shown in Fig. 4. Then, the 3D size distribution was derived by the Saltykov method and a step plot of pore size was created, as shown in Fig. 5. However, small negative numbers of pores were obtained for the 3D size distribution for the two smallest size bins $\left(N_{V}(13)<0\right.$ and $\left.N_{V}(14)<0\right)$ and were ignored. This is an inherent cumulative error for this stereological method, because some section areas of pores were too small to be distinguished during metallographic observation (Liu, 1999).

A Weibull distribution, density function denoted by $f(x)$ as follows, was used to fit the size distribution (Tobias and Trindade, 1995):

$f(x)=\frac{m}{x}\left(\frac{x}{c}\right)^{m} \mathrm{e}^{-(x / c)^{m}}$

where the parameters, $m$ and $c$, called shape and scale parameter respectively, must be positive. For 2D size distribution, by fitting the distribution of 2D pore size in Fig. $4, m=2.47$ and $c=64.68$ were obtained; for 3D size distribution, by fitting the distribution of 3D pore size in Fig. 5, $m=2.29$ and $c=60.52$ were obtained. The Weibull distributions

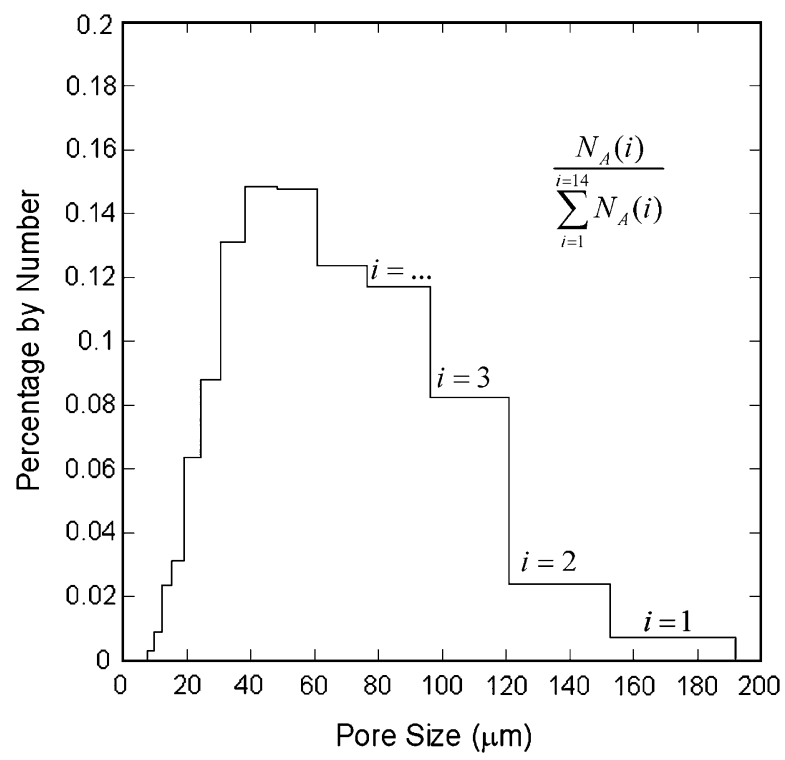

Fig. 4. Distribution of 2D pore sizes in titanium foam with $14.7 \%$ porosity. 


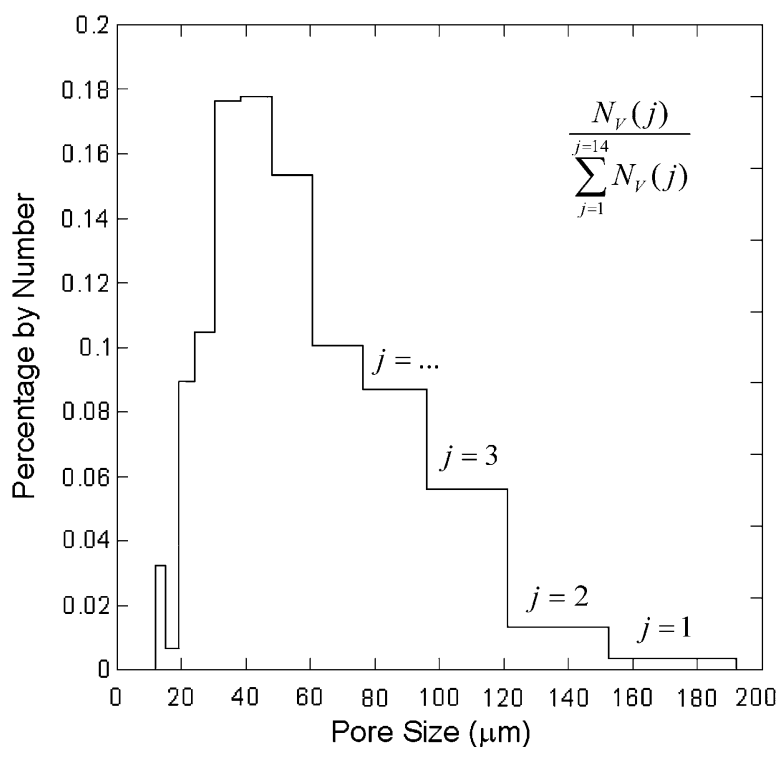

Fig. 5. Distribution of 3D pore size in titanium foam with $14.7 \%$ porosity derived by the Saltykov analysis.

of $2 \mathrm{D}$ and $3 \mathrm{D}$ pore size distributions are shown in Fig. 6. The average 3D pore size is somewhat lower than the average 2D pore section size. Initially, this result seems contradictory since $2 \mathrm{D}$ sections provide the intersection of pores with a metallographic sectioning plane and thus the 2D section of a pore will be less than or at the most equal to its true size in 3D microstructure. However, Louis and Gokhale

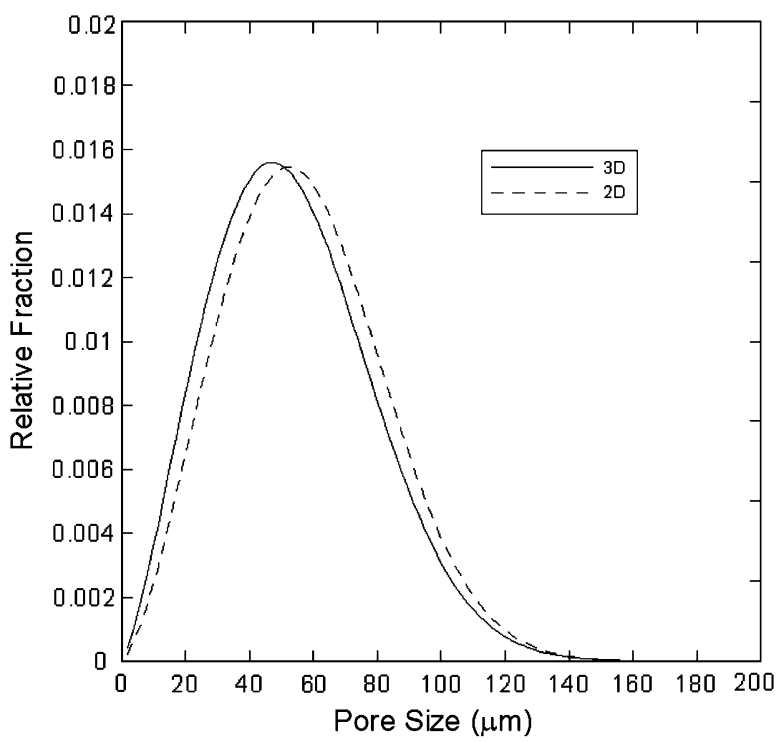

Fig. 6. Pore size distribution of $2 \mathrm{D}$ section and $3 \mathrm{D}$ reconstructed microstructure of titanium foam with $14.7 \%$ porosity, fit with a Weibull distribution function. (1995b) disproved this intuitive expectation of smaller average pore size for $2 \mathrm{D}$ sections. They derived the ratio of the average radius $\langle r\rangle$ of the pore sections observed in a $2 \mathrm{D}$ sectioning plane to the average sphere size $\langle R\rangle$ in a $3 \mathrm{D}$ microstructure to be

$\frac{\langle r\rangle}{\langle R\rangle}=\frac{\pi}{4}\left\{1+C V^{2}\right\}$

where $C V^{2}=\sigma^{2}(R) /[\langle R\rangle]^{2}$ and $\sigma^{2}(R)$ is the variance of sphere size distribution in the $3 \mathrm{D}$ microstructure. The wider the size distribution is, the larger the variance is and the higher $C V^{2}$ is. If $C V^{2}$ is larger than $[(4 / \pi)-1],\langle r\rangle$ is greater than $\langle R\rangle$. In our case $C V^{2}=0.281>[(4 / \pi)-1]$, which explains that the average $3 \mathrm{D}$ pore size is lower than the average $2 \mathrm{D}$ pore section size for the microstructure of the titanium foam.

\subsection{Location distribution of pores}

After powder densification but prior to pore expansion, pores in the densified titanium billet are very small (about $6 \mu \mathrm{m}$ in diameter) and occupy a very small area fraction (about $0.14 \%$ ) (Murray and Dunand, 2003). At this stage, the spatial distribution of the pores is not random, since they are located in the space between the prior titanium powder particles, which constitute an "excluded volume" for the pores. As pores grow during the foaming step and reach sizes comparable to those of the original titanium powders, the pore spatial distribution is expected to become closer to random. Since pore clustering has deleterious effects on the foam mechanical properties by creating local stress concentrations, it is important to determine how close to random the pore distribution is when pores have grown to sizes close to that of the initial powder size, as is the case for the titanium foam with $14.7 \%$ porosity shown in Fig. 2(a).

To quantify the randomness of the pore distribution, the $K$ function was used to process pore location data (Louis and Gokhale, 1995a). By drawing a circle of prescribed radius $R$ around the $i$ th pore, the total number of other pore centroids, $K^{i}(R)$, enclosed in the circle can be obtained. $K^{i}(R)$ depends on the location of the pore, the radius $R$, and the spatial arrangement of the pore centroids in the section of the specimen. The $K(R)$ function is the average value of $K^{i}(R)$ over all the pores in the section. Let $\bar{N}$ be the average number of pore centroids per unit area and $M$ be the total number of pores to be considered. The ratio 
Table 1

The pore distribution ratio, $f$, calculated from three different sections (one shown in Fig. 2(a)) and with three different $R$ values

\begin{tabular}{llllll}
\hline Section & Number of pores & Size (pixel) & $f(R=70$ pixel $)$ & $f(R=100$ pixel $)$ & $f(R=150$ pixel $)$ \\
\hline 1 & 2449 & $1218 \times 996$ & 0.998 & 1.012 & 1.004 \\
2 & 3089 & $1281 \times 1278$ & 0.980 & 0.992 & 0.998 \\
3 & 3096 & $1158 \times 1173$ & 0.976 & 0.987 & 1.001 \\
\hline
\end{tabular}

Results consistently imply a random distribution of pores for the sample at $14.7 \%$ porosity.

$f=\frac{K(R)}{\left[\left(\pi R^{2}\right) \bar{N}\right]}=\frac{\sum_{i=1}^{M} K^{i}(R)}{\left[M \cdot\left(\pi R^{2}\right) \bar{N}\right]}$

quantifies the pore distribution. $f=1$ signifies a random pore distribution, a higher value indicates a clustered distribution, and a lower value suggests pore segregation to surfaces of the model. The $K(R)$ function was then calculated on the large field section image obtained by the metallographic experiment in Fig. 2(a). As shown in Fig. 2(b), the centroids of pores in the section were used for the calculation. A circle with radius $R$ was drawn for each pore centroid within the section zone and the number of other pore centroids in the circle was counted. The section zone (dashed line in Fig. 2(b)) is inset by distance $\mathrm{R}$ from the edges of the full section to ensure that no circle falls outside the section. $\bar{N}$ is therefore calculated over the section zone area. Selecting three different circle radii for calculating $K(R)$ function, the ratio $f$ was calculated for three different sections and the results are listed in Table 1 . The values of $f$ are very close to unity, indicating that the pore distribution is nearrandom for the $14.7 \%$ volume fraction level shown in Fig. 2(a). It is noted that $\bar{N}$ was also calculated over several alternate area choices, such as over the whole section, and little variation of the values of $f$ were found.

\section{Reconstruction of the 3D microstructure}

An alternative method is needed to create the 3D porous microstructure of the target material at porosity of $40-50 \%$ due to the previously mentioned difficulty of using traditional techniques, such as serial sectioning methods, for such high porosity levels. The methodology proposed here first constructs a simulated microstructure of the titanium foam at low porosity combining simple 2D metallographic experimental data and statistic theory. The microstructure of the porous material at high porosity is then obtained by simulating pore growth and movement during foaming process.
For the present $14.7 \%$ titanium foam, the Weibull parameters of the $3 \mathrm{D}$ volumetric distribution of pores were derived and a computer program was developed to construct a representative $3 \mathrm{D}$ microstructure at low porosity. In the program, pores are sequentially generated in a $3 \mathrm{D}$ domain at random positions with their size following the Weibull random distribution with the parameters $m$ and $c$. After a given pore is created, the distance to all other existing pores is calculated. If intersection with these pores occurs, the created pore is discarded, a new pore is generated and the iteration is carried out again until no intersection is found. The porosity is monitored during the pore generation process and the process stops when the desired porosity is reached. Therefore, porosity and the Weibull parameter $m$ and $c$ are the key inputs for the program. Different size distribution of pores can be achieved by adjusting the Weibull parameters and a non-random distribution of pores can also be achieved by locating the distribution of pores with a different algorithm. Using $m=2.29$ and $c=60.52$ obtained in Section 3.1, a simulated microstructure for the target material with porosity of $14.7 \%$ was created.

To simulate the structure of materials foamed to high porosity, in previous work (Murray and Dunand, 2004b) the overall porosity development was determined using close-form solutions based on the pressure-vessel creep solution, or numerical FE 3D axisymmetric models with repeating boundary conditions. In these previous models, each pore was assumed to grow as a result of the internal gas pressure producing creep of the surrounding matrix. No pore coalescence was considered, so that the models can be considered valid for regular, periodic microstructures and porosities less than about $20 \%$. FE models for pore evolution to higher porosity with pore coalescence are under development. The goal of the present paper is to create simulated microstructures of high porosity that capture the key statistical geometric features of the target material in a full representative volume element for 
subsequent finite element analysis. Thus, a simpler approach is used and it is assumed that pores grow proportionally to achieve the desired higher porosity as explained below.

A computer program was written to use the $3 \mathrm{D}$ pore size and spatial distribution map from the previous step (at the low $14.7 \%$ porosity) and grow the pores to achieve a desired higher porosity. The size of the simulation is theoretically unlimited since the model is reconstructed using the continuous Weibull parameters, however, for practical reasons in terms of FE simulation time, model sizes of a $4000 \mu \mathrm{m}$ cube were typically chosen. During foaming, as in the experimental process, the cubic model expands, the pores grow and the pore locations also move proportionally (Davis, 2002). With volume expansion, the centroidal location of each pore moves proportionally to its distance from the origin:

$(x, y, z)_{f}^{p}=(x, y, z)_{0}^{p} \frac{L_{f}}{L_{0}}$

where $(x, y, z)_{f}^{p}$ are the final position coordinates of the $p$ th pore after the model is foamed to the desired porosity. $L_{0}$ and $L_{f}$ is the original and final length of the edge of the cube. Because the volume of solid is conserved, $L_{f}$ can be calculated from the original and target porosity. The pore growth is represented by

$V_{i}^{p}=V_{i-1}^{p} \frac{c_{\text {target }}}{c_{\text {current }}}$

where $V_{i}^{p}$ is the volume of the $p$ th pore at the $i$ th time-step, $c_{\text {target }}$ is the desired target porosity and $c_{\text {current }}$ is the current porosity. Thus, the ratio of the pore volumes before and after foaming are the same for each pore. The coalescence of pores is taken into account as illustrated in Fig. 7 where the coalescence of three pores is illustrated. Pores continue to grow after coalescence according to the above algorithm based on their original spherical shape: the changes in internal pore pressure with coalescence or the effects of nonuniform geometry are not yet accounted for here. Iteration is required to obtain the desired target porosity due to the pore coalescence, which excludes overlapping volume, and thus the model is run until the target porosity is achieved.

Software I-DEAS was then used as pre-processor to reconstruct the $3 \mathrm{D}$ microstructure of the low porosity and the foamed high porosity material. The data of pore sizes and centroids, reconstructed by the developed programs from the $2 \mathrm{D}$ metallographic data, were used as inputs to write scripts of I-DEAS. 3D finite element models were developed from which 2D models were obtained by cutting planar sections. Such 2D and 3D models are shown in Figs. 8 and 9. The size of the models is $1000 \mu \mathrm{m}$ and $1195.4 \mu \mathrm{m}$ on a side for 2D model in Fig. $8,340 \mu \mathrm{m}$ for 3D model with porosity of $14.7 \%$ in Fig. 9(a), and $680 \mu \mathrm{m}$ for 3D model with porosity of $50 \%$ in Fig. 9(b). These figures are qualitatively similar to reconstructed $3 \mathrm{D}$ structures of titanium foams with $38 \%$ porosity as shown in Fig. 9(c), acquired by synchrotron X-ray tomography (Murray et al., 2003).

Since the morphology of pores are influenced by many factors such as the size range of titanium powders and foaming parameters, this methodology for reconstructing microstructure of the foam is not predictive. However, this method produces reasonable 3D microstructures including coalesced pores and should provide superior simulation results for both local and global material response compared to $2 \mathrm{D}$ representations, $3 \mathrm{D}$ periodic microstructures or $3 \mathrm{D}$ random microstructures with non-interacting pores. In addition, by changing Weibull parameters of pore size distribution at certain porosity, the

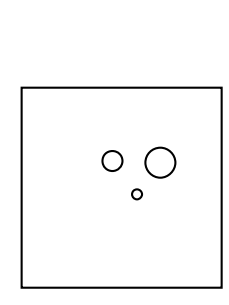

(a)

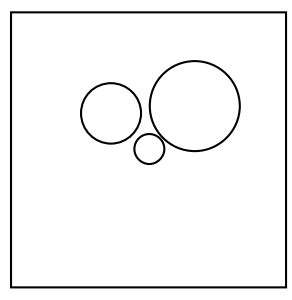

(b)

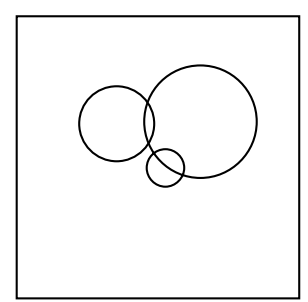

(c)

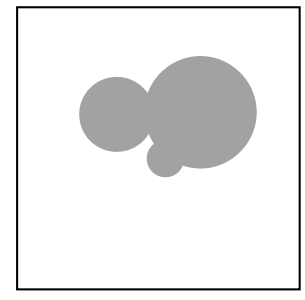

(d)

Fig. 7. Schematic of pore morphology evolution during foaming simulation: (a) material at low porosity; (b) pores grow and locations move due to expansion of the material during foaming (see text); (c) material is foamed to higher porosity and pores start to connect to each other; (d) pores coalesce to form a new large pore, excluding the overlapping volume. 


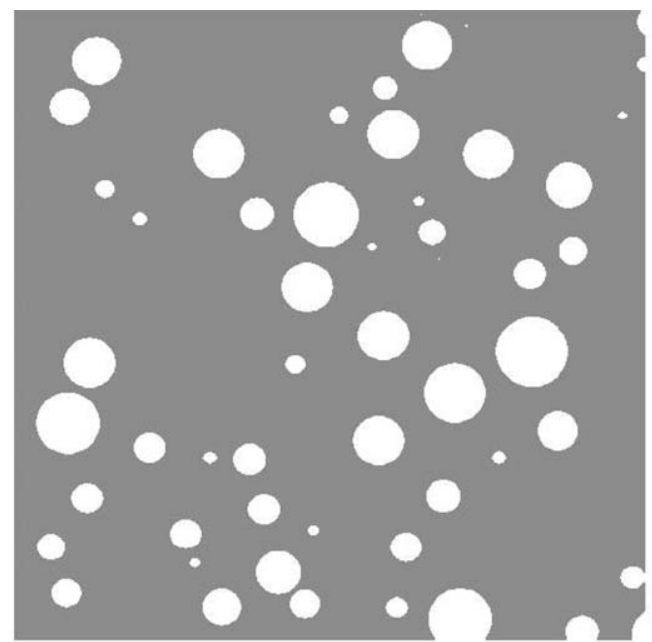

(a)

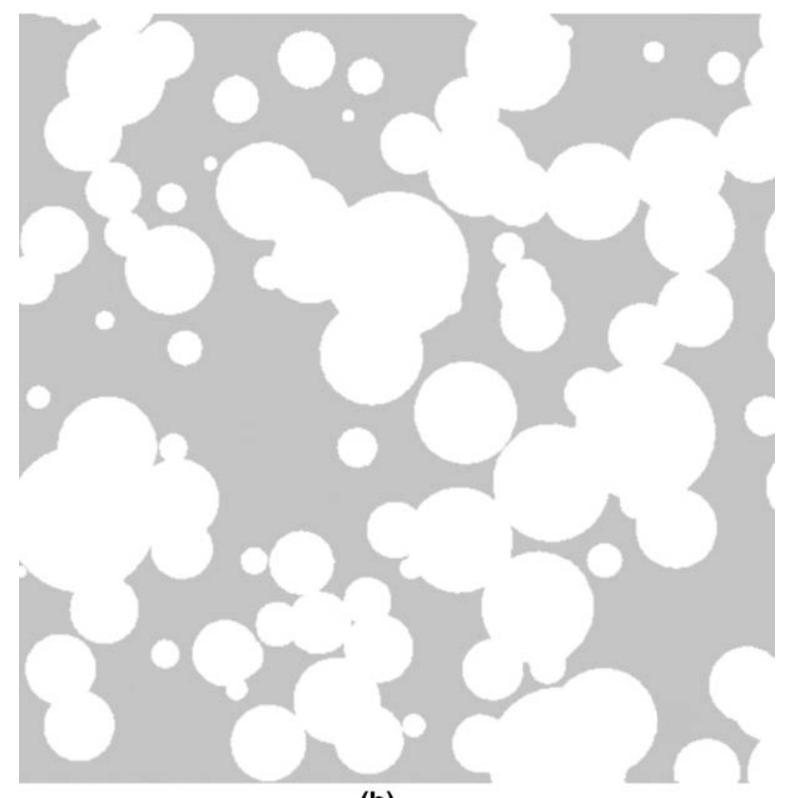

(b)

Fig. 8. Subsection of $2 \mathrm{D}$ simulated microstructure with a porosity of (a) $14.7 \%$ in a $1000 \mu \mathrm{m}$ square before the foaming process and (b) $52 \%$ in a $1195.4 \mu \mathrm{m}$ square after the simulated foaming process ((b) is the same section as (a) but after 3D foaming).

effect of pore size and clustering can be studied systematically.

\section{Finite element modeling}

For initial validation of the method, a 3D finite element analysis was carried out using the 3D model shown in Fig. 9(a). In the model, 66 pores were ran- domly distributed and the size distribution of the pores followed the characterization of the experimental material, providing a porosity of $14.7 \%$. Some pores were truncated since their centers are near a surface of the model. The pores and matrix were freely meshed with 129,232 10-node modified tetrahedral elements with hourglass control. The modified elements in ABAQUS exhibit minimum volumetric lock during plastic deformation (Hibbitt Karlsson Sorensen, Inc.). The mesh was verified to be fine enough by result convergence study. Boundary conditions were applied for the FE analysis, in which the four side faces remained straight and parallel during deformation, the bottom side was fixed in the normal direction, and a negative vertical displacement was applied on the top size to load the model to $1 \%$ compressive strain. Therefore, the boundary conditions are periodic mechanically, but not microstructurally as the pores on opposing edges are not continuous.

The computed macroscopic stress-strain response is shown in Fig. 10. Also shown in the figure is the constituent stress-strain response for the solid titanium matrix, using elastic modulus of $110 \mathrm{GPa}$, Poisson's ratio of 0.33 , and yield strength of $275 \mathrm{MPa}$ representative of CP-Ti-40 (ASM International, 2002). To avoid computational difficulties, it was assumed that pores were linear elastic with a very low modulus of $10^{-7} \mathrm{GPa}$, and Poisson's ratio of 0.3. A compressive experiment was performed on a specimen of titanium foam (with rectangular cross-section of $4.7 \mathrm{~mm} \times 4.6 \mathrm{~mm}$ and height of $9.0 \mathrm{~mm}$ ) with approximately $15 \%$ porosity and the experimental stress-strain curve is also shown in Fig. 10 for comparison. There were two loading and unloading loops before the specimen was loaded to a high strain. Strain accumulation can be observed at the end of the second loop. This residual strain might be caused by the imperfect boundary conditions of the experiment (friction between sample and platens) and/or plastic strain localization in the porous microstructure at low overall stress.

The 3D finite element model predicted Young's modulus of $82 \mathrm{GPa}$ and a $0.2 \%$ offset yield strength of $261 \mathrm{MPa}$. For comparison, the analytical prediction of Young's modulus by Gibson and Ashby is $80 \mathrm{GPa}$ (Gibson and Ashby, 1997). Based on the experiment, Young's modulus was found to be $78 \mathrm{GPa}$ by fitting the loading curve below $100 \mathrm{MPa}$ of the first loading segment, where minimal strain localization should have occurred. The 


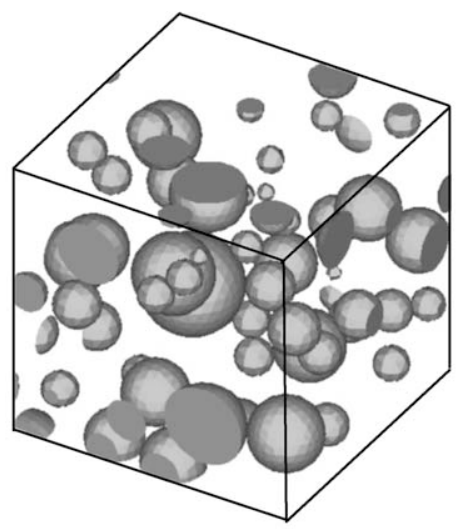

(a)

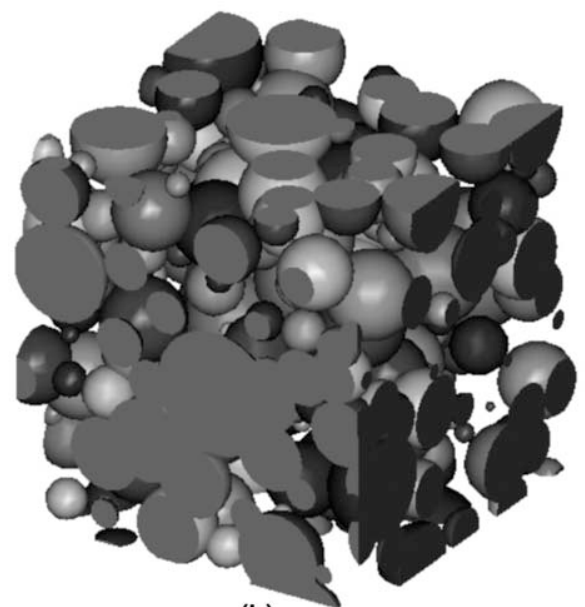

(b)

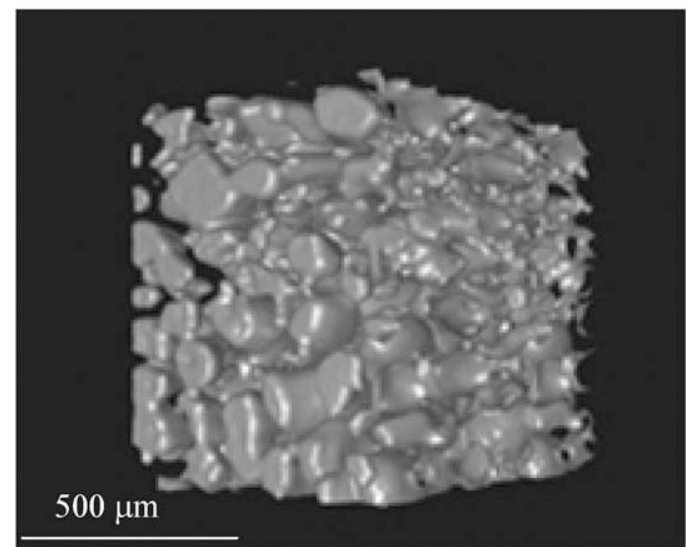

(c)

Fig. 9. (a) 3D finite element model with porosity of $14.7 \%$ in a $340 \mu \mathrm{m}$ cube, the small triangle facets on the pores are the mesh; (b) simulated microstructure of porous $\mathrm{Ti}$ with porosity of $50 \%$ in a $680 \mu \mathrm{m}$ cube; (c) reconstructed volume of titanium foam with $38 \%$ porosity, based on synchrotron X-ray tomographic data. Only the pore surfaces are shown for clarity (Murray et al., 2003).

$0.2 \%$ offset yield strength of the material is in rough agreement with the calculated value of $261 \mathrm{MPa}$. Thus, the predicted macroscopic responses from the FE analysis of the simulated 3D microstructure are in good agreement with the experimental data and theoretical results. However, it should be noted that only one sample was used for the experiment and it was reported by Davis et al. (2001) that there can be large scatter in experimental results for porous metals. Regarding the previous simulation works for porous material referred in Section 1, many of them, such as Li et al. (2004) and Segurado and Llorca (2002), did not compare simulation results with experimental data. While Sihn and Roy (2004) found a good agreement between their predictions with experimental data, their study only focused on elastic modulus. Thelen et al. (2004) found that 2D FE models underestimate the elastic modulus of titanium foam, and attributed the error to the inherent approximations in the $2 \mathrm{D}$ analysis.

In the current study, the size of the model to be analyzed as a representative volume element (RVE) was chosen to be $340 \mu \mathrm{m}$ on a side and the average pore diameter in the microstructure is $53.6 \mu \mathrm{m}$, making the model length to pore size ratio 6.34. While Drugan and Willis suggests two inclusion diameters at most are sufficient for an RVE, Ostoja-Starzewski's analysis shows that this ratio is problem-dependent (Drugan and Willis, 1996; Ostoja-Starzewski, 1998). When the inclusions are voids, even very large ratios (at least larger than $50)$ are inadequate. Jiang et al. $(2001,2002)$ and Bouyge et al. (2001, 2002) also suggested that RVE size determination for foam is still an unsolved 


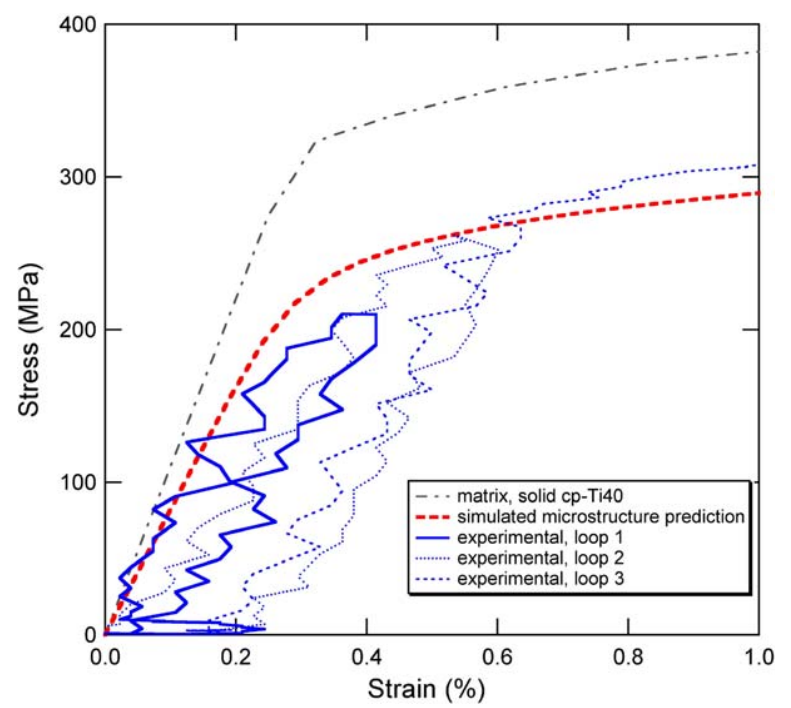

Fig. 10. Macroscopic stress-strain response of titanium foam obtained by $3 \mathrm{D}$ finite element analysis of the simulated $3 \mathrm{D}$ microstructure and a compressive test on a specimen of Ti foam at $15 \%$ porosity. Response of solid CP-Ti shown for reference.

problem. The RVE problem will become more significant for the target material with high porosity since the coalescence of pores creates larger pores and complicates the microstructure. This issue of RVE size is discussed thoroughly in companion papers (Shen et al., in preparation-a, in preparation-b). Both 2D and 3D finite element analysis are performed to study the mechanical behavior of the target foam at different porosities and the effect of local events on the global responses is investigated in detail in these related works.

\section{Conclusions}

A method has been created to simulate $3 \mathrm{D}$ porous microstructures of varying porosity based on minimal 2D microstructural information. A porous titanium material designed for bone implants was used to illustrate the procedure. Pore size and distribution in the material at low porosity were characterized by basic 2D metallography. Based on this information, the $3 \mathrm{D}$ volumetric distribution of pores was derived using stereology rules and a computer program developed to recreate the 3D microstructure based on this volume distribution function and the position distribution function (a random distribution was appropriate for the experimental material under study). To simulate a titanium foam at higher porosity, pore growth and coalescence was simulated in a separate program to generate a microstructure of the desired porosity level. A 3D finite element analysis has been performed for the material with porosity of $14.7 \%$. The stress-strain response was simulated and compares favorably with experimental results.

The method developed here has significant advantages over analysis of 3D tomographic approaches and simpler 2D reconstructed images. Obtaining 3D tomographic data is difficult in general and for foams in particular due to distortion during serial sectioning. While 2D micrographs can be more readily obtained and easily examined in FE, information on 2D sections can differ significantly from realistic 3D analyses, particularly for localized stress and deformation information. The current approach also represents a significant improvement over simulated 3D microstructures of periodic or random nonoverlapping pores and should provide more realistic analysis of local and global material response. In addition, the current analysis method can be used to understand the influence of some processing parameters on microstructure. As the initial porosity distribution depends on the size distribution and arrangement of the sieved powders, the Weibull parameters of pore size distribution and pore location distribution pattern can be altered to optimize these conditions for a desired 3D foamed microstructure.

\section{Acknowledgments}

The authors acknowledge the financial support of the National Science Foundation through grant number DMR-0108342 as well as the help and support from Dr. Yang Liu at GE Company. SMO also acknowledges the support of the Department of Defense through a NDSEG fellowship.

\section{References}

ASM International, 2002. Atlas of Stress-Strain Curves, 2nd ed. Benke, S., Weichert, D., 2003. Meso-mechanical modeling of damage in metal foams. Key Engineering Materials 251-252, 345-350.

Bouyge, F., Jasiuk, I., Boccara, S., Ostoja-Starzewski, M., 2002. A micromechanically based couple-stress model of an elastic orthotropic two-phase composite. European Journal of Mechanics A/Solids 21, 465-481.

Bouyge, F., Jasiuk, I., Ostoja-Starzewski, M., 2001. A micromechanically based couple-stress model of an elastic two-phase composite. International Journal of Solids and Structures 38, $1721-1735$ 
Chang, Y.-S., Oka, M., Kobayashi, M., Gu, H.-O., Li, Z.-L., Nakamura, T., Ikada, Y., 1996. Significance of interstitial bone ingrowth under load-bearing conditions: a comparison between solid and porous implant materials. Biomaterials 17, 1141-1148.

Davis, N.G., 2002. Enhancement of solid-state foaming of titanium by transformation superplasticity. Ph.D. Thesis.

Davis, N.G., Teisen, J., Schuh, C., Dunand, D.C., 2001. Solidstate foaming of titanium by superplastic expansion of argonfilled pores. Journal of Materials Research 16 (5), 15081519.

Drugan, W.J., Willis, J.R., 1996. A micromechanics-based nonlocal constitutive equation and estimates of representative volume element size for elastic composites. Journal of the Mechanics and Physics of Solids 44 (4), 497-524.

Dunand, D.C., 2004. Processing of titanium foams. Advanced Engineering Materials 6 (6), 369-376.

Gibson, L., Ashby, M., 1997. Cellular Solids: Structure and Properties, Second ed. Cambridge University Press, Cambridge, UK.

Hibbitt Karlsson Sorensen Inc., 2004. ABAQUS Ver. 6-4 Theory Manual.

Jiang, M., Jasiuk, I., Ostoja-Starzewski, M., 2002. Apparent elastic and elastoplastic behavior of periodic composites. International Journal of Solids and Structures 39, 199 212.

Jiang, M., Ostoja-Starzewski, M., Jasiuk, I., 2001. Scale-dependent bounds on effective elastoplastic response of random composites. Journal of the Mechanics and Physics of Solids 49, 655-673.

Li, H., Oppenheimer, S.M., Stupp, S.I., Dunand, D.C., Brinson, L.C., 2004. Effects of pore morphology and bone ingrowth on mechanical properties of microporous titanium as an orthopaedic implant material. Materials Transactions 45 (4), 1124 1131.

Li, M., Ghosh, S., Rouns, T.N., Weiland, H., Richmond, O., Hunt, W., 1998. Serial sectioning method in the construction of 3-D microstructures for particle-reinforced MMCs. Materials Characterization 41, 81-95.

Li, M., Ghosh, S., Richmond, O., Weiland, H., Rouns, T.N., 1999. Three dimensional characterization and modeling of particle reinforced metal matrix composites. Part I: Quantitative description of microstructural morphology. Materials Science and Engineering A265, 153-173.

Liu, Y., 1999. Three dimensional grain size distribution and grain structure reconstruction in liquid phase sintering. Ph.D. Thesis.

Liu, Y., German, R.M., Iacocca, R.G., 1999. Microstructure quantification procedures in liquid-phase sintered materials. Acta Materialia 47 (3), 915-926.

Long, M., Rack, H.J., 1998. Titanium alloys in total joint replacement $-\mathrm{a}$ materials science perspective. Biomaterials $19,1621-1639$.

Louis, P., Gokhale, A.M., 1995a. Application of image analysis for characterization of spatial arrangements of features in microstructure. Metallurgical and Materials Transactions A 26A, 1449-1456.

Louis, P., Gokhale, A.M., 1995b. Can the average particle section size in a metallographic plane be larger than the true average particle size in a three-dimensional microstructure. Metallurgical and Materials Transactions A 26A, 1741-1744.
McDanels, D., 1985. Analysis of stress-strain, fracture, and ductility behavior of aluminum matrix composites containing discontinuous silicon carbide reinforcement. Metallurgical Transactions A 16A, 1105-1115.

Murray, N.G.D., Dunand, D.C., 2004a. Effect of thermal history on the superplastic expansion of argon-filled pores in titanium. Part I: Kinetics and microstructure. Acta Materialia 52 (8), 2269-2278

Murray, N.G.D., Dunand, D.C., 2004b. Effect of thermal history on the superplastic expansion of argon-filled pores in titanium. Part II: Modeling of kinetics. Acta Materialia 52 (8), 2279-2291.

Murray, N.G.D., Dunand, D.C., 2003. Microstructure evolution during solid-state foaming of titanium. Composites Science and Technology 63, 2311-2316.

Murray, N.G.D., Schuh, C.A., Dunand, D.C., 2003. Solid-state foaming of titanium by hydrogen-induced internal-stress plasticity. Scripta Materialia 49 (9), 879-883.

Okazaki, Y., Rao, S., Tateishi, T., Ito, Y., 1998. Cytocompatibility of various metal and development of new titanium alloys for medical implants. Materials Science and Engineering A243, 250-256.

Ostoja-Starzewski, M., 1998. Random field models of heterogeneous materials. International Journal of Solids and Structures 35, 2429-2455.

Segurado, J., Llorca, J., 2002. A numerical approximation to the elastic properties of sphere-reinforced composites. Journal of the Mechanics and Physics of Solids 50, 2107-2121.

Shan, Z., Gokhale, A.M., 2001. Micromechanics of complex three-dimensional microstructures. Acta Materialia 49, 20012015.

Shen, H., Lissenden, C.J., 2002. 3D finite element analysis of particle-reinforced aluminum. Material Science and Engineering A338, 271-281.

Shen, H., Oppenheimer, S.M., Dunand, D.C., Brinson, L.C., in preparation-a. Finite element modeling of titanium foam as an orthopaedic implant.

Shen, H., Oppenheimer, S.M., Dunand, D.C., Brinson, L.C., in preparation-b. A numerical investigation of the effect of boundary conditions and RVE size on simulation of foamed titanium.

Sihn, S., Roy, A.K., 2004. Modeling and prediction of bulk properties of open-cell carbon foam. Journal of Mechanics and Physics of Solids 52, 167-191.

Spoerke, E.D., Murray, N.G., Li, H., Brinson, L.C., Dunand, D.C., Stupp, S.I., in press. A bioactive titanium foam scaffold for bone repair. Acta Biomaterialia.

Thelen, S., Barthelat, F., Brinson, L.C., 2004. Mechanics considerations for microporous titanium as an orthopaedic implant material. Journal of Biomedical Materials Research 69A, 601-610.

Tobias, P.A., Trindade, D.C., 1995. Applied Reliability, 81-104. Underwood, E.E., 1970. Quantitative stereology. pp. 109-147.

Wen, C.E., Yamada, Y., Shimojima, K., Chino, Y., Asahina, T., Mabuchi, M., 2002a. Processing and mechanical properties of autogenous titanium implant materials. Journal of Materials Science: Materials in Medicine 13, 397-401.

Wen, C.E., Yamada, Y., Shimojima, K., Chino, Y., Hosokawa, T., Mabuchi, M., 2002b. Novel titanium foam for bone tissue engineering. Journal of Materials Research 17 (10), 2633 2639 . 